\title{
Rendimiento académico en escuelas de nivel medio superior
}

\author{
Estefanía Gordillo Rico \\ Javier Martínez Morales \\ Herik Germán Valles Baca \\ Profesores investigadores \\ Facultad de Economía Internacional de la \\ Universidad Autónoma de Chihuahua
}

\section{Resumen}

E

n el presente trabajo se hace un análisis descriptivo sobre los resultados de una encuesta realizada a estudiantes de cuatro instituciones de educación media superior de la ciudad de Hidalgo del Parral, Chihuahua: ConAlep plantel Parral, Colegio de Bachilleres No. 12, Preparatoria Ramón López Velarde y el Свтіs 228, para valorar qué variables están relacionadas con el rendimiento académico del alumnado. Los principales resultados señalan que leer más de un libro al año, estar en turno matutino, vivir con los padres y tener buena relación con ellos, implica un mejor rendimiento académico, mientras que trabajar y estudiar de manera simultánea y consumir algún tipo de droga están asociadas con un bajo desempeño académico.
Palabras clave: Rendimiento académico, consumo de sustancias nocivas, género, bachillerato.

\section{Introducción}

La educación es uno de los factores más relevantes para las sociedades contemporáneas. La literatura ha señalado que los efectos de tener poblaciones educadas generan un mejor y mayor nivel de desarrollo económico. Sin embargo, también se ha encontrado que alumnas y alumnos con bajo desempeño académico pueden generar deserción, desigualdad educativa, reprobación entre otros.

Desde una perspectiva de política pública, un bajo rendimiento académico puede implicar que el alumno está recibiendo una formación académica de baja calidad o de- 
ficiente. Por ello, es importante analizar la situación en la que se encuentran las y los alumnos con referencia a su rendimiento académico.

El rendimiento académico es un fenómeno multifocal. Edel (2003) explica que el rendimiento académico debe centralizarse en el esfuerzo y la habilidad del estudiante. Sin embargo, la importancia de éste radica en cómo se relaciona con otras variables. Por ejemplo: calificaciones, niveles de inteligencia e incluso con niveles socioeconómicos y socioculturales. Tejedor (2003) define al rendimiento como inmediato si éste se relaciona con las calificaciones que logran los alumnos a través de su carrera profesional hasta obtener un título.

Por otro lado Torres y Rodríguez (2006) señalan que el rendimiento académico consiste en alcanzar un nivel de conocimientos que se evidencie en un área o materia, confrontado con su edad y nivel académico o con la norma. Vicente (2000) demuestra que las propiedades psicométricas de las calificaciones escolares son un instrumento eficaz y eficiente para ser ocupadas como medición del rendimiento académico.

Es importante mencionar que el rendimiento académico se vincula con resultados palpables, que evidencien de cierta forma que la o el estudiante tienen como referencia ese conocimiento adquirido, finalmente lo que interesa a un empleador durante los procesos de selección es la demostración de ese conocimiento a través de notas o calificaciones de los estudiantes (Ocaña, 2011). Gutiérrez y Montañez (2012) proponen que el rendimiento académico sea el reflejo de la obtención de conocimientos que adquiere un alumno o alumna de un grado educativo a través de la institución educativa, este nivel cognitivo que la escuela otorga al estudiante puede ser expresado mediante una calificación.

Para Edel (2003) el rendimiento académico tiene una amplia complejidad y variedad de definiciones; sin embargo, afirma que esta gama de enunciados solo se explican por cuestiones de semántica. Se puede decir, que en efecto existe un debate en la definición de rendimiento académico, pero para fines de este documento, al hablar de rendimiento académico se hace referencia al promedio del ciclo inmediato anterior cursado por el o la alumna encuestada.

Pero ¿qué elementos están asociados al rendimiento académico de las y los estudiantes? Para responder esta pregunta diversos trabajos muestran la relación de los factores que alteran al desempeño o rendimiento académico. Beltrán y La Serna (2009) encuentran que el rendimiento académico del bachillerato tiene una fuerte asociación con el rendimiento académico durante toda la carrera universitaria.

Torres y Rodríguez (2006) sostienen que el rendimiento académico tiene una relación positiva cuando los estudiantes tienen acceso a libros, copias, material didáctico, lugar y tiempo para estudiar, pero una relación negativa con los problemas familiares, maestros y clases, la distancia y el transporte. Zorrilla y Muro (2004) evidencian que el rendimiento académico tiene una vinculación positiva con el contexto familiar, si es mujer, el grado en que se encuentre cursando la secundaria y si está en el turno matutino. 
Córdoba (2011) enfatiza que el rendimiento académico del alumno depende positivamente de una familia que tenga papá, mamá y hermanos, pero negativamente si la familia está desestructurada y de un bajo nivel educativo y económico de los padres. Aunado al trabajo anterior, Caso y Hernández (2007) muestran que la autoestima y las habilidades de estudio tienen efecto positivo en el rendimiento académico y de forma negativa, con el consumo de sustancias nocivas para la salud, sin embargo no encuentra evidencia suficiente para analizar el efecto de asertividad, establecimiento de metas y la adaptación escolar.

Para efectos de este trabajo, se hace un análisis descriptivo sobre los resultados de la encuesta realizada en cuatro instituciones de educación media superior de Hidalgo del Parral, Chihuahua. El propósito es mostrar la relación del rendimiento académico con promedio de calificaciones del semestre inmediato anterior, con variables que la literatura ha considerado que guardan una relación positiva o negativa.

Estos resultados nos permitirán proponer en una segunda etapa: un modelo de impacto de las variables socioeconómicas, culturales y pedagógicas sobre el rendimiento académico y generar un segundo modelo que buscará hacer referencia al análisis de la probabilidad de qué factores afectan a los alumnos de alto y bajo rendimiento académico.

\section{Estrategia Metodológica}

\section{Población y muestra}

La población que se consideró para este trabajo son alumnos y alumnas de preparatoria entre 15 y 18 años que estudian en Hidalgo del Parral, un total de 4,187 estudiantes de acuerdo con los datos recabados en las diferentes instituciones de educación media superior.

Se consideraron las cuatro escuelas de educación media superior más representativas, por el número de estudiantes que atienden en el municipio de Parral. Las escuelas son: el Colegio de Bachilleres No. 12, la escuela Ramón López Velarde, el Colegio Nacional de Educación Profesional Técnica Plantel Parral (ConAleP) y el Centro de Bachillerato Tecnológico Industrial y de Servicios 228 (Свтіs 228). La encuesta se realizó durante el semestre agosto-diciembre de 2012 y por tanto sólo se consideró a alumnas y alumnos del segundo, cuarto y sexto semestres. El cuadro I presenta un resumen de las escuelas consideradas en el estudio así como el alumnado en los turnos matutino y vespertino.

\begin{tabular}{|l|l|l|l|}
\hline \multicolumn{3}{|c|}{ Cuadro I. Instituciones Educativas de Media Superior } \\
\hline Institución Educativa & Matutino & Vespertino & $\begin{array}{l}\text { Total de } \\
\text { alumnos }\end{array}$ \\
\hline Ramón López Velarde & 556 & 386 & 947 \\
\hline CBTIS 228 & 1006 & 769 & 1775 \\
\hline COBACh & 451 & 251 & 705 \\
\hline CONALEP & 527 & 241 & 768 \\
\hline TOTAL & 2540 & 1647 & 4187 \\
\hline
\end{tabular}


Para calcular el tamaño de la muestra se consideró lo siguiente:

- El porcentaje de confianza con el cual se quiere generalizar los datos desde la muestra hacia la población total.

- El porcentaje de error que se pretende aceptar al momento de hacer la generalización.

- El porcentaje de grado de confianza es de 95\%, para el error se aceptó entre $4 \%$ y $6 \%$, tomando en cuenta que son complementarios la confianza y el error. De lo anterior se desprende la siguiente fórmula.

$$
n=\frac{Z^{2} p q N}{N E^{2}+Z^{2} p q}
$$

Dónde:

n es el tamaño de la muestra

$\mathrm{Z}$ es el nivel de confianza

p es la variabilidad positiva

q es la variabilidad negativa

$\mathrm{N}$ es el tamaño de la población

E es la precisión o el error.
Por lo cual

$$
\mathrm{N}=4187 \text { Estudiantes }
$$

$Z=$ la confianza es del $95 \%$, es decir, el intervalo de confianza es $\mathrm{P}(-1.96<\mathrm{z}<1.96)$

$$
\begin{aligned}
& p=0.5 \\
& q=0.5 \\
& E=5 \%
\end{aligned}
$$

$$
\begin{gathered}
n=\frac{(1.96)^{2}(0.5)(0.5)(4187)}{(4187)(0.05)^{2}+(1.96)^{2}(0.5)(0.5)} \\
n=\frac{4021.19}{11.42} \\
n=352.118
\end{gathered}
$$

Se aplicaron 352 cuestionarios, los cuales fueron distribuidas en las cuatro instituciones educativas seleccionadas en turno matutino y vespertino. La encuesta consistió en 23 preguntas que fueron elaboradas con base en los perfiles que se describen en el cuadro II. 
Cuadro II. Perfiles para análisis de rendimiento académico

\begin{tabular}{|c|c|c|c|}
\hline Perfiles & Indicadores básicos & Perfiles & Indicadores básicos \\
\hline $\begin{array}{l}\text { Personal y } \\
\text { Situación } \\
\text { Académica }\end{array}$ & $\begin{array}{l}\text { - Género } \\
\text { - Edad } \\
\text { - Escuela } \\
\text { - Semestre } \\
\text { - Grupo } \\
\text { - Promedio del semestre anterior } \\
\text { - Turno }\end{array}$ & Socioeconómico & $\begin{array}{l}\text { El alumno vive con: padre, mamá, } \\
\text { amigos, solo, pareja, ambos padres, otro. } \\
\text { - Escolaridad de los padres } \\
\text { - Dependencia económica } \\
\text { - Ingreso familiar mensual } \\
\text { - Servicios domésticos } \\
\text { - Transporte } \\
\text { - Ocupación del padre y de la madre }\end{array}$ \\
\hline $\begin{array}{l}\text { Contexto } \\
\text { Familiar }\end{array}$ & $\begin{array}{l}\text { - Relación familiar } \\
\text { - Apoyo familiar } \\
\text { - Exigencia de buenas } \\
\text { calificaciones } \\
\text { - Lectura } \\
\text { - Influencia familiar }\end{array}$ & Situación Laboral & $\begin{array}{l}\text { - Trabajo, horas dedicadas } \\
\text { - Tiempo de trabajo } \\
\text { - Flexibilidad de trabajo }\end{array}$ \\
\hline $\begin{array}{l}\text { Situación de } \\
\text { Riesgo }\end{array}$ & $\begin{array}{l}\text { - Consumo de sustancias nocivas } \\
\text { para la salud } \\
\text { - Frecuencia de consumo; } \\
\text { 1. Tabaco } \\
\text { 2. Alcohol } \\
\text { 3. Cocaína } \\
\text { 4. Marihuana } \\
\text { 5. Alucinógenos } \\
\text { 6. Inhalables } \\
\text { - Ofrecimiento de droga; edad }\end{array}$ & & \\
\hline
\end{tabular}

\section{Principales resultados}

De total de las y los encuestados, 145 fueron hombres y 207 mujeres. Las edades oscilaron entre los 15 y 21 años de edad. El rendimiento académico tuvo una media de 8.38 , que en general es un promedio aceptable. Desde la perspectiva de género el promedio de las mujeres fue de 8.49 mientras que el de los hombres alcanzó un 8.24. El cuadro III muestra los promedios por escuela y por sexo.

Dentro de los datos obtenidos se clasificó a los alumnos en alto y bajo rendimiento académico. El cuadro IV presen-

\begin{tabular}{|c|c|c|c|}
\hline \multicolumn{4}{|c}{ Cuadro III. Rendimiento académico } \\
\hline Escuela & Total & Mujeres & Hombres \\
\hline CBTIS & 8.37 & 8.46 & 8.20 \\
\hline COBACh & 8.26 & 8.58 & 8.04 \\
\hline CONALEP & 8.80 & 8.89 & 8.61 \\
\hline Ramón López Velarde & 8.19 & 8.29 & 8.12 \\
\hline TOTAL & 8.39 & 8.49 & 8.24 \\
\hline
\end{tabular}
ta los resultados.

\begin{tabular}{|c|c|c|c|}
\hline \multicolumn{4}{|c|}{ Cuadro IV. Desempeño } \\
\hline Rendimiento & Hombres & Mujeres & Total \\
\hline Bajo & 34 & 53 & 87 \\
\hline Alto & 111 & 154 & 265 \\
\hline Total & 145 & 207 & 352 \\
\hline
\end{tabular}


En el perfil socioeconómico se encontró que el $68 \%$ de los encuestados vive con los padres, mientras que el $2 \%$ vive solo. Otro resultado interesante es la escolaridad del padre, ésta se mide por años acumulados de estudio, el $30.4 \%$ tiene preparatoria completa, seguido de secundaria completa con $29.8 \%$ y para el caso de la madre, el $30.4 \%$ tiene secundaria completa mientras que el 29.5\% tiene preparatoria completa. Como se observa, la educación tanto del padre como de la madre es muy similar.

Sin duda el ingreso de las familias también es un gran soporte para que el alumnado continúe el estudio y es considerado como factor que contribuye a un buen rendimiento. Los datos muestran que el $44 \%$ de las familias ganan de $\$ 1,650.00$ a $\$ 4,750.00$ pesos mensuales, mientras que el $32 \%$ gana de $\$ 4,751.00$ a $\$ 8250.00$ pesos mensuales. La ocupación del padre es mayoritariamente comerciante, seguido de profesionistas y técnicos, sin embargo 32\% de las madres son amas de casa, cifra muy elevada si comparamos su grado de escolaridad.

El apoyo de la familia desde el punto de vista moral siempre es parte fundamental para un mejor rendimiento académico. Los resultados de la encuesta reflejan que el $82 \%$ de las y los encuestados respondió tener total apoyo de la familia para sus estudios, un $51 \%$ respondió que sus padres les exigen estudiar y un $47 \%$ respondió que cree que sus padres influyen en su aprendizaje. El 51\% de los estudiantes también respondió que lee de uno a dos libros por año, sin embargo el $32 \%$ respondió que no lee ningún libro por año.
Dentro del perfil laboral, el 78\% de los estudiantes no trabaja, seguido de un 22\% sí lo hace. Dentro de ese 22\%, el 45\% lleva empleado un máximo de 11 meses y el 79\% le dedica de una a siete horas por semana al trabajo. Esto es un reflejo de la flexibilidad del trabajo para seguir estudiando.

A continuación se muestra el perfil de situación de riesgo, es decir, el consumo de sustancias nocivas para la salud de parte del estudiantado.

En muchas ocasiones la situación de riesgo implica un empeoramiento en el rendimiento académico. Los resultados muestran que el $45 \%$ de las y los estudiantes encuestados consumen algún tipo de sustancia nociva para la salud, llámese tabaco, alcohol o algún tipo de droga. De ese $45 \%$, el $54 \%$ corresponde a hombres y el $46 \%$ a mujeres, cifra que llama la atención ya que no hay mucha diferencia en los porcentajes entre hombres y mujeres. Al mismo tiempo se tiene que ese $45 \%$, el $31 \%$ a menudo fuma cigarro, sin embargo se agrava con el consumo de alcohol llegando a hacerlo muy a menudo el $60 \%$ de los estudiantes. El 6\% del total del 45\%, que consumen sustancia nociva, consume a menudo cocaína y el $9.6 \%$ consume marihuana. El $68 \%$ de la muestra total respondió que alguna vez le han ofrecido algún tipo de droga ilegal. Pero ¿qué escuela consume más sustancias nocivas para la salud? De acuerdo con las respuestas del alumnado, en la figura I se muestran los porcentajes obtenidos. Como puede observarse las escuelas con mayor porcentaje de alumnos que consumen alguna sustancia nociva, legal o ilegal, son el CBTIS, seguido del COBACH. 
Lo que interesa es observar la relación que tiene el rendimiento académico entre las variables antes mencionadas. El cuadro $\mathrm{V}$ muestra un resumen de los principales resultados. Para entender el cuadro, se presentan el nombre de las variables y a la derecha de ellas se muestra el rendimiento académico que se obtiene. En la mayoría de las variables se observa las relaciones positivas que ya han mostrado algunos otros autores (Torres y Rodríguez, 2006, Armenta, Pacheco y Pineda, 2008; Torres, 2011); sin embargo, llama la atención la vinculación que guarda la escolaridad tanto del padre con el rendimiento académico de los hijos; esto es, a medida que se incrementa la escolaridad del padre el rendimiento académico de los hijos no siempre mejora, peor aún: a medida que crece la escolaridad de la madre el rendimiento académico del hijo tiende a caer; fenómeno que requiere estudiarse de forma más profunda. Otra variable que también requiere análisis es que a medida que se incrementa el ingreso familiar el rendimiento tiende a caer.

Hasta aquí se puede observar que tener buenas relaciones con la familia y leer más de un libro al año tiene un efecto positivo sobre el rendimiento académico de las y los alumnos. Como era de esperarse el consumo de drogas, alcohol o tabaco genera una caída del rendimiento académico.

\section{Conclusiones}

Los resultados muestran dos puntos importantes: el primero de ellos es que la estadística descriptiva permite dar un pa- norama general sobre la problemática del rendimiento académico de los y las alumnas de las escuelas de Hidalgo del Parral. El hecho de leer algún libro al año, tener una buena relación con los padres, estar en el turno matutino y vivir con los padres, refleja un mejor rendimiento académico o aprovechamiento; por otro lado, si el alumno trabaja, sus padres tienen un ingreso superior a los 8,250 pesos o se consume algún tipo de droga, cualquiera que sea, implica un menor rendimiento académico. Aunque la escolaridad del padre parece tener un nulo efecto sobre el rendimiento y el de la madre tiene un efecto negativo.

El segundo punto a tratar es que la estadística descriptiva no permite generar por sí sola un análisis de impacto sobre el rendimiento académico, es decir, no se puede adelantar al hecho de si estas variables tienen un efecto significativo o no. Por lo cual, no podemos generar políticas públicas para el mejoramiento del rendimiento académico de los y las alumnas.

Al mismo tiempo este trabajo tiene una perspectiva meramente del lado de la demanda más no de la oferta, es decir, hace falta profundizar en las características de las escuelas, profesorado y medios de aprendizaje dentro de las escuelas.

Queda pendiente el estudio de modelos de impacto y probabilísticos que serán analizados en una segunda etapa del análisis del rendimiento académico de las y los alumnos en los bachilleratos de la ciudad de Hidalgo del Parral. 


\section{Bibliografía}

Armento, N.G., Pacheco, C., Pineda, E.D. (2008). Factores socioeconómicos que intervienen en el desempeño académico de los estudiantes de la Facultad de Ciencias Humanas de la Universidad Autónoma de Baja California. Recuperado el día 13 de abril de 2013 en http://sisbib.unmsm.edu. pe/bvrevistas/investigacion_psicologia/v11_n1/pdf/a10.pdf

BeLtrán, A., La Serna, K. (2009). ¿Qué explica la evolución del rendimiento académico universitario? Un estudio de caso en la Universidad del Pacifico. Recuperado el día 4 de febrero de 2013 de http://www. up.edu.pe/ciup/SiteAssets/ Lists/JER_Jerarquia/EditForm/20091214225310_DD-09-15.pdf

Caso, J., Hernández, L. (2007). Variables que inciden en el rendimiento académico de adolescentes mexicanos. Recuperado el día 13 de abril de 2013 en http:// www.redalyc.org/articulo.oa?id $=80539304$

Córdoba, L.G., García, V., Luengo. L.M., Vizuete, M. y Feu, S. (2011). Determinantes socioculturales: Su relación con el rendimiento académico en alumnos de enseñanza secundaria obligatoria. Recuperado el día 13 de abril de 2013 de http://www.redalyc.org/articulo.oa?id=283322813005

Edel, R. (2003). El Rendimiento Académico: Concepto, Investigación y Desarrollo. Recuperado el día 13 abril del 2013 de http://www.ice.deusto.es/RINACE/reice/vol1n2/Res_Edel.htm

Gutiérrez, S., Montañez, G.S. (2012). Análisis teórico sobre el concepto de rendimiento académico escolar y la influencia de factores socioculturales. Recuperado el día 10 de mayo de 2012 de http://www.ride. org.mx/docs/publicaciones/09/ practica_educativa/ Sandra_Gutierrez_Olvera-loria_Silviana_Montanez_Moya.pdf
OCAÑA, Y. (2011). Variables académicas que influyen en el rendimiento académico de los estudiantes universitarios. Recuperado el día 13 de abril de 2013 de http://sisbib.unmsm.edu.pe/bibvirtual/publicaciones/inv_educativa/2011_n27/a11v15n27.pdf

Tejedor, F.J. (2003). Poder explicativo de algunos determinantes del rendimiento en los estudios universitarios. Recuperado el día 13 de abril del 2013 de http://reforma.fen.uchile.cl/Papers/Poder\%20explicativo\%20de\%20los\%20determinantes\%20del\%20 rendimiento\%20en\%20los\%20estudios\%20universitarios\%20-\%20Tejedor.pdf

Torres, L.E., Rodríguez, N.Y. (2006). Rendimiento académico y contexto familiar en estudiantes universitarios. Recuperado el día 13 de abril del 2013 de http:// www.redalyc.org/articulo.oa?id=29211204

Torres, L.E. (2011). Rendimiento académico, familia y equidad de género. Recuperado el día 2 de febrero de 2013 de http://www.redalyc.org/articulo. oa? id=87019755003

Vicente, I. (2000). Análisis de las calificaciones escolares como criterio de rendimiento académico. Recuperado el 28 de abril de 2013 de http://campus.usal. es/ inico/investigacion/jornadas/jornada2/comun/ c17.html

Zorrilla, M., Muro, F.J. (2004). La enseñanza secundaria en México 2002. Una exploración de modelos explicativos de resultados de aprendizaje y características del alumno, del entorno familiar y escolar. Recuperado el día 20 de mayo de 2013 de http://www.oei. es/evaluacioneducativa/exploracion_modelos_explicativos_resultados_aprendizaje_mexico_zorrilla. pdf. 